\title{
Hiperlactatemia à Admissão na UTI é Um Determinante de Morbimortalidade em Intervenções Cirúrgicas não Cardíacas de Alto Risco*
}

\author{
Hyperlactatemia at ICU Admission is a Morbid-Mortality \\ Determinant in High Risk Non-Cardiac Surgeries
}

\author{
Samantha L. S. Almeida ${ }^{1}$, Cristina P. Amendola ${ }^{1}$, Vanessa M. Horta ${ }^{1}$, Érica Sousa ${ }^{2}$, \\ Célio A. B. Gusmão ${ }^{2}$, João M. Silva Júnior ${ }^{3}$, Ederlon Rezende ${ }^{4}$
}

\section{RESUMO}

JUSTIFICATIVA E OBJETIVOS: Um dos maiores desafios dos médicos intensivistas é o controle da hipoperfusão tecidual, sendo o lactato sérico classicamente aceito como indicador de hipóxia tecidual. Deste modo, estudos demonstraram boa correlação entre o lactato e o prognóstico no choque e durante a reanimação. O objetivo deste estudo foi avaliar a utilidade clínica do lactato arterial à admissão na UTI como indicador de morbimortalidade em pacientes críticos no pós-operatório de intervenções cirúrgicas não cardíacas de alto risco.

MÉTODO: Estudo de coorte prospectivo observacional, realizado em UTI de hospital terciário no período

1. Residente em Medicina Intensiva do Serviço de Terapia Intensiva Hospital do Servidor Público Estadual.

2. Médico Assistente do Serviço de Terapia Intensiva Hospital do Servidor Público Estadual.

3. Médico Supervisor do Serviço de Terapia Intensiva Hospital do Servidor Público Estadual.

4. Diretor do Serviço de Terapia Intensiva do Hospital do Servidor Público Estadual.

*Recebido do Serviço de Terapia Intensiva do Hospital do Servidor Público Estadual Francisco Morato de Oliveira (HSPE-FMO), São Paulo, SP

- Apresentado pela Dra. Samantha L. S. Almeida para obtenção do Título de Especialista em Medicina Intensiva pela Associação de Medicina Intensiva Brasileira - AMIB.

Apresentado em 11 de julho de 2006

Aceito para publicação em 16 de novembro de 2006

Endereço para correspondência:

Rua Pedro de Toledo, $18006^{\circ}$ andar - Vila Clementino

04039-901 São Paulo, SP

Fone/Fax (11) 5088-8146

E-mail: ederlon@sti-hspe.com.br

(CAssociação de Medicina Intensiva Brasileira, 2006 de 4 meses. Foram coletados dados demográficos, lactato arterial e complicações no pós-operatório de pacientes submetidos à intervenções cirúrgicas de grande porte. Para análise estatística foi considerado significativo $p<0,05$. A habilidade preditiva dos índices em diferenciar sobreviventes e não sobreviventes foi verificada utilizando curvas ROC. Estimativas de permanência na UTI foram calculadas utilizando o método de Kaplan Méier.

RESULTADOS: Foram incluídos 202 pacientes, sendo $50,2 \%$ do sexo feminino, com média da idade de 66,5 \pm 13,6 anos, APACHE II 17,4 $\pm 3,0$, mediana do MODS 4 (2-6). A duração mediana das intervenções foram $4 \mathrm{~h}$ (3 a 6 h), $70,7 \%$ de cirurgias eletivas, a mortalidade na UTI e hospitalar foram $15,6 \%$ e $33,7 \%$, respectivamente. $O$ melhor valor de lactato que discriminou mortalidade foi $3,2 \mathrm{mmol} / \mathrm{L}$, sensibilidade de $62,5 \%$ e especificidade de $78,8 \%$, área sob a curva de 0,7 . Não sobreviveram $62,5 \%$ dos pacientes com lactato $\geq 3,2$ versus $21,2 \%$ de sobreviventes (OR = 2,95 IC95\% 1,98 - 4,38, p < 0,0001). O tempo de permanência na UTI foi mais elevado quando lactato $\geq 3,2 \mathrm{mmol} / \mathrm{L}$ (log rank 0,007 ).

CONCLUSÕES: Os pacientes cirúrgicos não cardíacos de alto risco admitidos na UTI com hiperlactatemia, definida com lactato $\geq 3,2 \mathrm{mmol} / \mathrm{L}$, apresentaram risco aumentado de morte e permanência prolongada na UTI.

Unitermos: lactato, morbidade, mortalidade, pacientes cirúrgicos.

\section{SUMMARY}

BACKGROUND AND OBJECTIVES: One of the greatest challenges found by the intensivists in their daily activities is tissue hipoperfusion control. Blood lactate is generally accepted as a marker of tissular hypoxia and 
several studies have demonstrated good correlation between blood lactate and prognosis during shock and resuscitation. The aim of this study was to evaluate the clinical utility of arterial blood lactate as a marker of morbidity and mortality in critically ill patients in the postoperative period of high risk non-cardiac surgeries.

METHODS: Prospective and observational cohort study realized in an ICU of a tertiary hospital during a four month period. Demographic data of the patients submitted to high risk surgeries were collected, besides arterial lactate measures and number and type of complications in the post-operative period. To the statistic analysis was considered as significant a $p<0.05$. The predictive ability of the indexes to differentiate survivors from non-survivors was tested using ROC curves. Lenght of ICU stay estimation where calculated by Kaplan Meier method.

RESULTS: Were included 202 patients. 50.2\% were female and their mean age was $66.5 \pm 13.6$ years. APACHE II score was $17.4 \pm 3.0$ and the median of MODS score was 4 (2-6). Median lenght of surgeries was $4 \mathrm{~h}$ (3-6h). $70.7 \%$ of the surgeries were elective ones. ICU and hospital mortality were $15.6 \%$ and $33.7 \%$, respectively. The best lactate value to discriminate mortality was $3.2 \mathrm{mmol} / \mathrm{L}$, with sensitivity of $62.5 \%$, specificity of $78.8 \%$ and an area under the curve of $0.7 .62 .5 \%$ of patients with lactate $\geq 3.2$ did not survive versus $21.2 \%$ of survivors (OR $=2.95 \mathrm{IC} 95 \%$ 1.98- 4.38, $\mathrm{p}<0.0001)$. ICU lenght of stay was greater when $\geq 3.2 \mathrm{mmol} / \mathrm{L}$ (log rank 0.007) lactate.

CONCLUSIONS: High risk patients submitted to non cardiac surgeries and admitted to the ICU with hiperlactatemia, defined as an arterial lactate $\geq 3.2 \mathrm{mmol} / \mathrm{L}$, are prone to a longer ICU lenght of stay and to die.

Key Words: lactate, morbidity, mortality, surgical patients

\section{INTRODUÇÃO}

O número de pacientes cirúrgicos internados em unidades de terapia intensiva (UTI) é na maioria das vezes predominante. Mais de 40 milhões de intervenções cirúrgicas são realizadas anualmente nos EUA e na Inglaterra. Sendo que, alguns destes milhões, devem-se a procedimentos cirúrgicos de moderado a alto risco. A mortalidade observada em pacientes de alto risco varia de 9,7\% nos EUA a 35,9\% na Inglaterra. O desfecho cirúrgico destes pacientes é influenciado pelo estado fisiológico pré-operatório, pelo risco cirúrgico e pelo adequado cuidado no pós-operatório1.
Assim, a importância de dados preditivos de risco para morbidade e mortalidade, para este grupo, torna-se fundamental ${ }^{2}$, sendo a hipoperfusão uma das causas.

A hipoperfusão aguda pode ser caracterizada por um desequilíbrio entre a oferta e o consumo de oxigênio pelos tecidos, o que proporciona uma falha em suprir as necessidades metabólicas, culminando em alto risco de múltiplas disfunções orgânicas ${ }^{3,4}$. Um importante indicador de hipoperfusão é o lactato, e este vem sendo estudado desde a década de 1960 por Weil e Afifi ${ }^{5}$, mostrando que concentrações séricas elevadas na admissão está fortemente associada ao prognóstico dos pacientes ${ }^{6}$. O lactato é produto do metabolismo anaeróbico onde ocorre conversão de piruvato em lactato pela catalisação da enzima lactato desidrogenase e quando encontrado em altas concentrações séricas, torna-se um preditor de mortalidade e morbidade relacionado com disfunções múltiplas de órgãos ${ }^{7}$.

Em determinadas situações como na sepse, no trauma e no choque o uso da dosagem do lactato sérico como indicador de hipóxia tecidual está estabelecido ${ }^{7-9}$. No entanto, existem poucos dados com relação aos pacientes cirúrgicos ${ }^{10}$. Waxman e col. estudaram concentrações de lactato durante e após intervenções cirúrgicas, onde os níveis pressóricos e o débito cardíaco não se alteravam, mas havia elevação do lactato ${ }^{11}$.

Tradicionalmente, a normalização de sinais vitais, como a pressão arterial sistêmica, débito urinário e freqüência cardíaca, têm sido usadas como meta de reanimação, mas atualmente com estudos sobre a microcirculação e perfusão, tais parâmetros tornaram-se inadequados $3,12,13$.

O objetivo deste estudo foi avaliar a utilidade clínica do lactato arterial à admissão na UTI como indicador de morbimortalidade em pacientes críticos no pós-operatório de intervenções cirúrgicas não cardíacas de alto risco.

\section{MÉTODO}

Estudo de coorte prospectivo observacional realizado durante período de 01 de abril a 31 de julho de 2005, em Unidade de Terapia Intensiva com 20 leitos de hospital público terciário de São Paulo, onde os cuidados foram realizados por equipe multidisciplinar composta por médicos intensivista plantonista e diarista. Foram coletados dados consecutivos de 202 pacientes após aprovação pelo Comitê de Ética 
e Pesquisa, que dispensou o termo de consentimento livre e esclarecido.

Foram incluídos no estudo todos os pacientes submetidos à intervenções cirúrgicas de grande porte, os quais apresentavam grande exposição da cavidade abdominal ou torácica por mais de duas horas, grande sangramento - necessitando transfusão de sangue ou alguma repercussão sistêmica, com idade superior a 18 anos e encaminhados para pós-operatório na UTI, exceto pacientes de cirurgia cardíaca.

Foram coletados os piores valores das variáveis nas primeiras 24 horas da admissão na UTI, para a realização de escores APACHE II (Acute Physiology and Chronic Healt Evalution II) ${ }^{14-16}$ e MODS (Multiple Organ Dysfunction Score $)^{2}$, outros dados como análise demográfica, tipo de cirurgia (eletiva ou urgência), tempos de cirurgia, internação na UTI e hospitalar, juntamente com a coleta do lactato arterial foram avaliados durante os pós-operatórios imediato, que compreendia desde a admissão na UTI até às 6 horas do dia seguinte. Os pacientes foram acompanhados até alta ou óbito na UTI. O pesquisador não exercia qualquer influência nas decisões dos médicos que assistiam o paciente.

Rotineiramente neste serviço de terapia intensiva, os médicos assistentes têm como critério de má perfusão tecidual a hiperlactatemia e as metas de reanimação são atingidas com a normalização do lactato sérico arterial.

Para análise estatística, os dados foram demonstrados como média \pm DP, mediana (intervalo interquartil) e porcentagens, para analise das variáveis com distribuição normal foi utilizado o teste $t$ de Student, variáveis com distribuição irregular o teste de Mann-Withney e variáveis categóricas o teste Qui-quadrado, com o valor de $\mathrm{p}<0,05$ (2-tailed) considerado significativo. A habilidade preditiva dos índices em diferenciar sobreviventes e não sobreviventes foi verificada utilizando curvas ROC (Receiver Operating Characteristic). Estimativas de curvas de permanência na UTI foram calculadas utilizando o método de Kaplan Méier com as diferenças verificadas através do teste logaritmo de escores (log rank test).

\section{RESULTADOS}

Foram analisados 202 pacientes durante quatro meses, sendo $50,2 \%$ do sexo feminino e média de idade de 66,5 \pm 13,6 anos, com APACHE II de 17,4 $\pm 6,0$ e o MODS de 40 (2-6). Houve prevalência das cirurgias eletivas $(70,7 \%)$ e do aparelho digestivo $(46,9 \%)$, a sua duração foi 4h (3 a 6h). A mortalidade hospitalar e na UTI foram $33,7 \%$ e $15,6 \%$, respectivamente. A mediana do lactato arterial foi de $2,2(1,6-3,5)$ (Tabela 1$)$.

Tabela 1 - Características dos Pacientes

\begin{tabular}{lc}
\hline Características & Variáveis \\
\hline Cirurgia eletiva & $70,7 \%$ \\
Idade (anos) & $66,5 \pm 13,6$ \\
Sexo feminino & $50,2 \%$ \\
APACHE II & $17,4 \pm 6,0$ \\
MODS & $4,0(2,0-6,0)$ \\
Duração da cirurgia (horas) & $4,0(3,0-6,0)$ \\
Internação na UTI (dias) & $1(1,0-3,0)$ \\
Internação no hospital (dias) & $17,0(9-33)$ \\
Internação prévia à cirurgia (dias) & $6(3-13)$ \\
Freqüência cardíaca (bpm) & $109,8 \pm 22,4$ \\
Pressão arterial média (mmHg) & $71,5 \pm 16,2$ \\
Hematócrito (\%) & $32,2 \pm 6,5$ \\
Lactato arterial (mmol/L) & $2,2(1,6-3,5)$ \\
Mortalidade na UTI & $15,6 \%$ \\
Mortalidade no hospital & $33,7 \%$ \\
\hline
\end{tabular}

O valor de lactado de $3,2 \mathrm{mmol} / \mathrm{L}$ apresentou melhor sensibilidade $(62,5 \%)$ e especificidade $(78,8 \%)$ para mortalidade na UTI, com área sob a curva de 0,7 (Figura 1). $O$ valor de lactato arterial $\geq 3,2 \mathrm{mmol} / \mathrm{L}$ foi então chamado de hiperlactatemia.

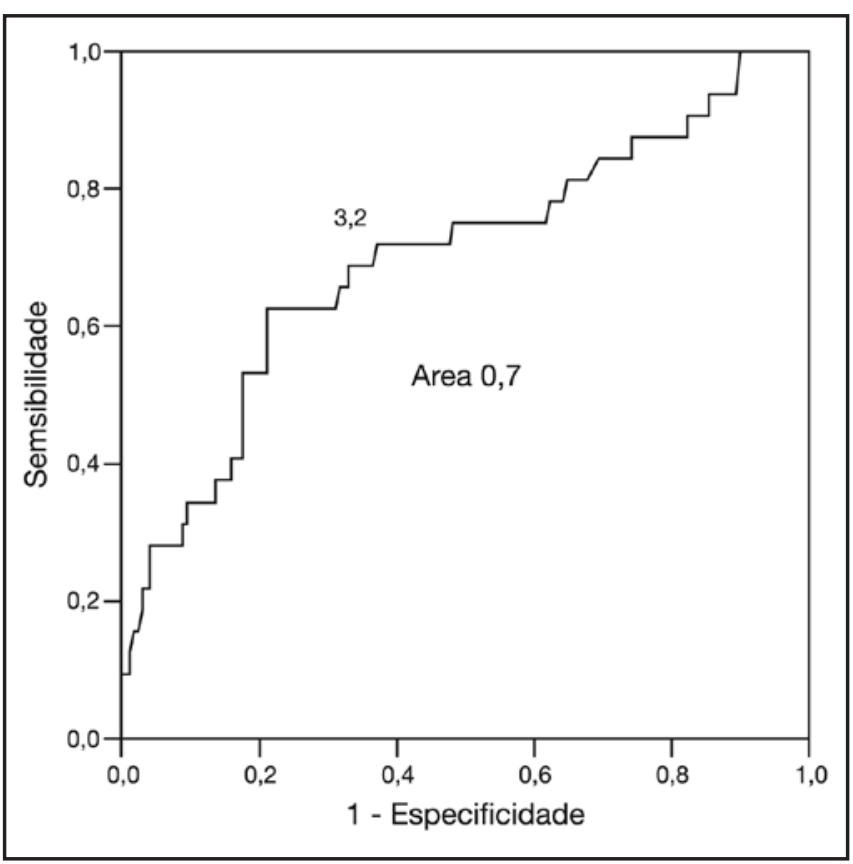

Figura 1 - Curva ROC para Mortalidade na UTI 
Não sobreviveram $62,5 \%$ dos pacientes com lactato maior ou igual a 3,2 versus $21,2 \%$ de sobreviventes $(\mathrm{OR}=2,95 \mathrm{IC} 95 \%$ 1,98 - 4,38, $\mathrm{p}<0,0001)$ (Figura 2).

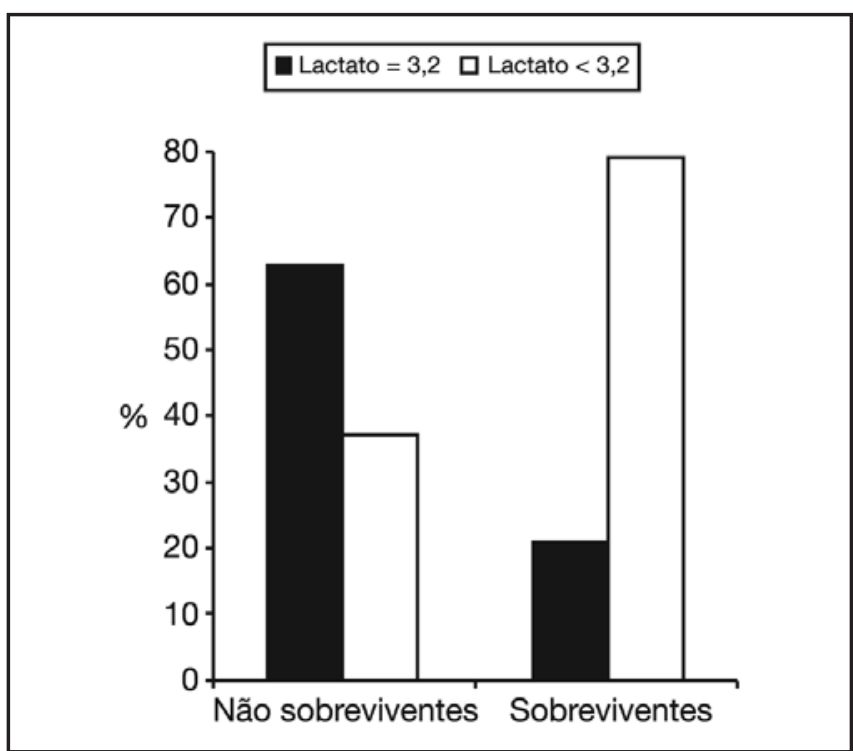

Figura 2 - Porcentagem de Sobreviventes ou não Sobreviventes para Pacientes com lactato $\geq 3,2 \mathrm{mmol} / \mathrm{L}$ e lactato $<3,2$ $\mathrm{mmol} / \mathrm{L}$.

Quando comparados os sobreviventes e os não sobreviventes, a mediana do lactato foi de $2,0(0,5-8,6)$ e 3,6 (1-18), respectivamente (Figura 3).

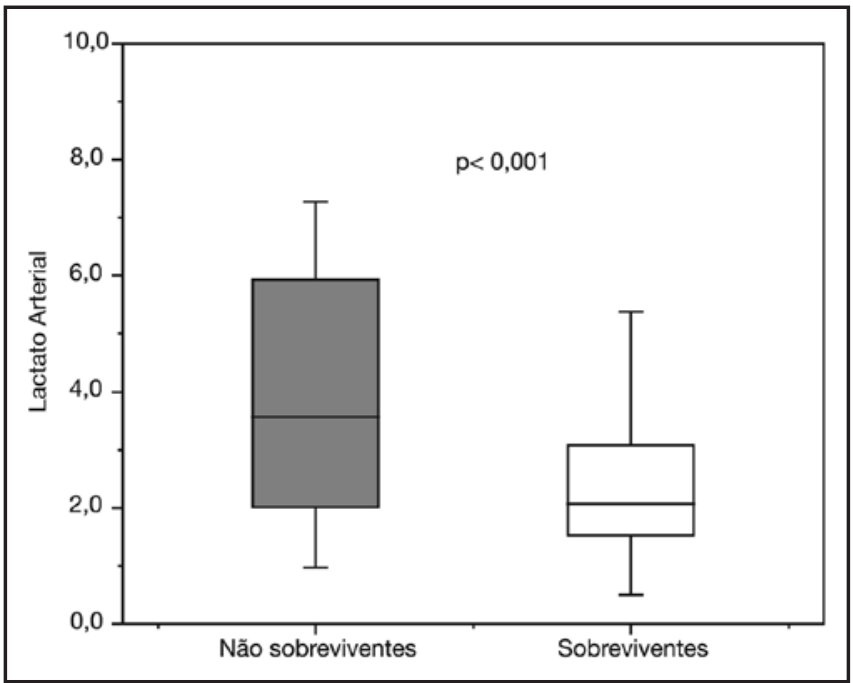

Figura 3 - Valor Mediano do Lactato para Alta ou Óbito

Comparando os grupos com e sem hiperlactatemia, foram significativos os escores APACHE II e MODS (Tabela 2).
Tabela 2 - Comparação entre os Pacientes com e sem Hiperlactatemia

\begin{tabular}{lccc}
\hline Variáveis & Lactato $<3,2(146)$ & Lactato $\geq 3,2(56)$ & $\mathrm{p}$ \\
\hline Idade & $65,7 \pm 14,1$ & $68,5 \pm 11,6$ & 0,2 \\
APACHE II & $16,5 \pm 5,5$ & $20,1 \pm 6,4$ & $0,001^{*}$ \\
MODS & $4,0 \pm 2,5$ & $5,6 \pm 3,1$ & $0,001^{*}$ \\
Tempo de ci- & $4,8 \pm 2,6$ & $4,6 \pm 2,3$ & 0,66 \\
rurgia & & & \\
Glicemia & $149,7 \pm 56,7$ & $165,7 \pm 60,1$ & 0,07 \\
Cirurgia de ur- & 28,1 & 30,4 & 0,75 \\
gência (\%) & & & \\
Sexo feminino & 48,6 & 53,6 & 0,53 \\
(\%) & & & \\
\hline
\end{tabular}

* $p$ significativo

Os pacientes com hiperlactatemia apresentaram maior tempo de internação na UTI (Figura 4).

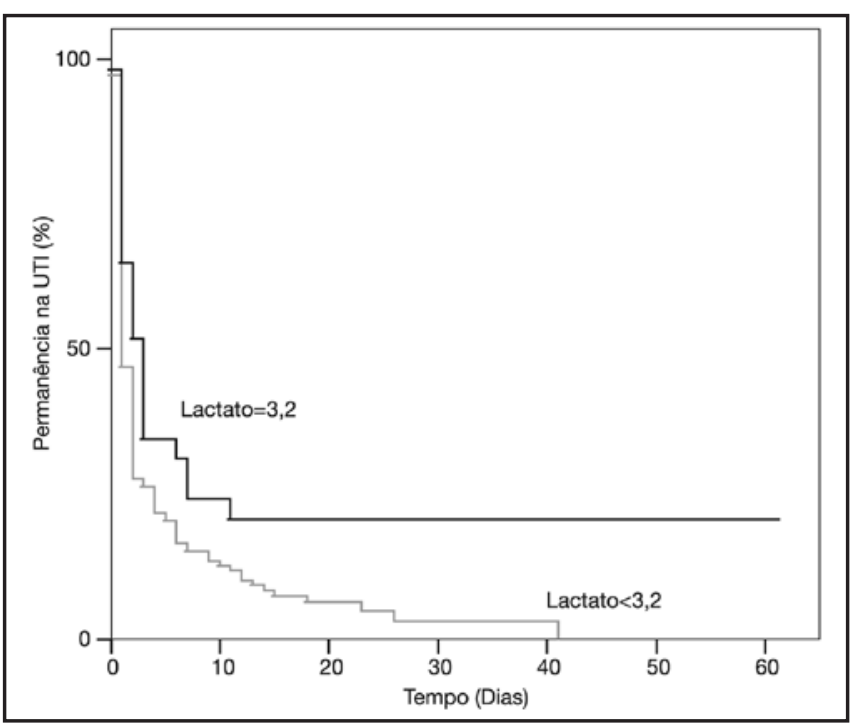

Figura 4 - Curva de Permanência Hospitalar em Pacientes com Lactato $\geq 3,2$ e $<3,2$

\section{DISCUSSÃO}

O presente estudo, com 202 pacientes selecionados, apresentaram dados já observados de lactato como indicador prognóstico num grupo pouco estudado; o de pacientes no pós-operatório de intervenções cirúrgicas não cardíacas de alto risco.

$\mathrm{Na}$ amostra observada, o número de cirurgias eletivas foi maior $(70,7 \%)$ comparado às de urgência. $O$ que traz maior confiabilidade dos resultados apresentados, sem o viés das cirurgias de urgência, onde as condições clínicas e cirúrgicas são inadequadas e, conseqüentemente, a existência de maiores taxas de morbidade e mortalidade na UTI e no hospital. Dessa forma, neste estudo, a evolução pós-operatória dos pacientes 
submetidos à intervenções cirúrgicas eletivas está intimamente relacionada com o seu manuseio no intraoperatório.

A duração das cirurgias foi de $4 \mathrm{~h}$ ( 3 a $6 \mathrm{~h}$ ), com mortalidade hospitalar de $33,7 \%$ mostrando o alto risco cirúrgico dessa população, equiparando-se com a mortalidade em pacientes de alto risco cirúrgico na Inglaterra que é de $35,9 \%{ }^{1}$. A semelhança do modelo brasileiro ao inglês deve-se também as condições de pouca disponibilidade de leitos de UTI, dinâmica de internação prévia à cirurgia e alta hospitalar, alta gravidade da população desencadeada pelo baixo investimento do país em leitos, equipamentos, tratamento e equipe especializada de UTI. Na Inglaterra esse gasto gira em torno de $1 \%$ a $3 \%$ em contraste com $20 \%$ a $34 \%$ nos EUA, onde a mortalidade dessa população fica em $9,7 \%{ }^{1}$.

Dessa forma, um dos maiores desafios dos médicos intensivistas é evitar a alta mortalidade e a progressão para múltiplas disfunções, controlando a hipoperfusão tecidual neste grupo ${ }^{18}$, sendo o lactato sérico já demonstrado em diversos estudos $3,5,7,8$ um bom indicador de hipóxia tecidual e prognóstico.

No estudo em questão foi encontrado o valor de lactato de $3,2 \mathrm{mmol} / \mathrm{L}$, como o que melhor discriminou mortalidade em pacientes cirúrgicos não cardíacos de alto risco, comparado a literatura, com $3,1 \mathrm{mmol} / \mathrm{L} \mathrm{em}$ pacientes de cirurgias não cardíacas ${ }^{3}$ e $3 \mathrm{mmol} / \mathrm{L}$ de cirurgias cardíacas ${ }^{19}$. Meregalli e col. ${ }^{3}$ mostraram que o valor de lactato de 3,1 $\mathrm{mmol} / \mathrm{L}$ em pacientes cirúrgicos, hemodinamicamente estáveis no pós-operatório discriminaram, de forma não significativa, sobreviventes de não sobreviventes nas primeiras 12 horas da admissão, porém somente o não clareamento desse valor foi que determinou pior prognóstico. Este como outros autores ${ }^{17,20}$ relacionaram a hiperlactatemia em pacientes hemodinamicamente estáveis como estado de hipoperfusão oculta. Em cirurgias cardíacas o valor do lactato de $3 \mathrm{mmol} / \mathrm{L}$ no pós-operatório determinou risco aumentado de morbidade e mortalidade ${ }^{18}$.

Smith e col..$^{21} \mathrm{em}$ estudo onde $50 \%$ dos pacientes foram cirúrgicos, observaram que o aumento dos níveis séricos de lactato na admissão apresentava forte relação com a mortalidade.

Nos pacientes com sepse o ponto de corte para determinar pior prognóstico, ficou ao redor de $2 \mathrm{mmol} / \mathrm{L}^{22}$, o que difere do estudo apresentado com concentrações mais elevadas.

Durante os procedimentos cirúrgicos, principalmente em cirurgias de grande porte, ocorre maior utilização de fármacos vasoativos e diminuição da depuração hepática, pela diminuição do metabolismo celular proporcionado pela anestesia. Por esse motivo, pacientes no pós-operatório imediato apresentam valores de lactado sérico mais elevados, justificado pela utilização de aminas vasoativas, pois com a administração de catecolaminas o metabolismo da glicose excede a capacidade oxidativa da mitocôndria e pelo wash out após anestesia, com o restabelecimento da função metabólica celular ${ }^{23}$.

Os índices APACHE II e MODS são excelentes indicadores prognósticos em pacientes cirúrgicos ${ }^{24,25}$, confirmando os resultados deste estudo. Estes se assemeIharam com o lactato. Portanto, o lactato arterial $\geq 3,2$ $\mathrm{mmol} / \mathrm{L}$ da chegada do paciente na UTI, mostrou ser o parâmetro mais prático e de fácil realização à beira leito, que os complexos cálculos matemáticos para determinar a evolução dos pacientes.

Outras variáveis como idade, sexo, tempo e tipo de cirurgia não mostraram correlação com o lactado elevado e o desfecho na UTI.

Entretanto, ao contrário de outros estudos $3,26-28$, esta análise não procurou verificar a normalização do lactado durante a evolução dos pacientes, o que poderia melhorar a acurácia do método, tornando os resultados encontrados mais completos. Assim como, também não foi avaliada a prática de reanimação realizada pelos médicos intensivistas frente à hiperlactatemia na admissão destes pacientes na UTI, poderia de alguma forma ter impacto na sobrevida.

Em adição, a hiperlactatemia nem sempre significa pior prognóstico e hipoperfusão ${ }^{29}$, podendo estar presente em situações clínicas como a insuficiência hepática ${ }^{22}$, por haver diminuição da sua excreção, distúrbio da piruvato desidrogenase, alcalose respiratória, administração de catecolaminas, porém o fato não invalida o método como excelente indicador de perfusão tecidual.

A mensuração do lactado arterial no pós-operatório imediato de intervenções cirúrgicas não cardíacas de alto risco mostrou ser um bom indicador de morbidade e mortalidade na evolução dos pacientes.

\section{REFERÊNCIAS}

01. Bennett-Guerrero E, Hyam JA, Shaefi S et al - Comparison of P-POSSUM risk-adjusted mortality rates after surgery between patients in the USA and the UK. Br J Surg, 2003;90:1593-1598.

02. Marshall JC, Cook DJ, Christou NV et al - Multiple organ dysfunction score: a reliable descriptor of a complex clinical outcome. Crit Care Med, 1995;23:1638-1652.

03. Meregalli A, Oliveira RP, Friedman G - Occult hypoperfusion is associated with increased mortality in hemodynamically stable, high-risk, surgi- 
cal patients. Crit Care, 2004;8:R60-R65.

04. De Backer D - Lactic acidosis. Minerva Anestesiol, 2003;69:281-284.

05. Weil MH, Afifi AA - Experimental and clinical studies on lactate and pyruvate as indicators of the severity of acute circulatory failure (shock). Circulation, 1970;41:989-1001.

06. Bakker J, Lima A - Monitorização da Perfusão Tecidual, em: Rea NA, Mendes CL, Rezende EAC et al - Monitorização em UTI. $1^{\text {a }}$ Ed, Rio de Janeiro, Revinter, 2004;89-101.

07. Rivers E, Nguyen B, Havstad S et al - Early goal-directed therapy in the treatment of severe sepsis and septic shock. $\mathrm{N}$ Engl J Med, 2001;345:1368-1377.

08. Bakker J, Coffernils M, Leon M et al - Blood lactate levels are superior to oxygen-derived variables in predicting outcome in human septic shock. Chest, 1991;99:956-962.

09. Husain FA, Martin MJ, Mullenix PS et al - Serum lactate and base deficit as predictors of mortality and morbidity. Am J Surg, 2003;185:485-491.

10. Bakker J, de Lima AP - Increased blood lactate levels: an important warning signal in surgical practice. Crit Care, 2004;8:96-98.

11. Waxman K, Nolan LS, Shoemaker WC - Sequential perioperative lactate determination. Physiological and clinical implications. Crit Care Med, 1982;10:96-99.

12. Blow O, Magliore L, Claridge JA et al - The golden hour and silver day: detection and correction of occult hypoperfusion within 24 hours improves outcome from major trauma. J Trauma, 1999;47:964-969.

13. Scalea TM, Maltz S, Yelom J et al - Resuscitation of multiple trauma and head injury: role of crystalloid and inotropes. Crit Care Med, 1994;22:1610-1615.

14. Knaus WA, Zimmerman JE, Wagner DP et al - APACHE-acute physiology and chronic health evaluation: a physiologically based classification system. Crit Care Med, 1981;9:591-597.

15. Knaus WA, Draper EA, Wagner DP et al - APACHE II: a severity of disease classification system. Crit Care Med, 1985;13:818-829.

16. Knaus WA, Wagner DP, Draper EA et al - The APACHE III prognostic system. Risk prediction of hospital mortality for critically ill hospitalized adults. Chest, 1991;100:1619-1636.

17. Blow O, Magliore L, Claridge JA et al - The golden hour and the silver day: detection and correction of occult hypoperfusion within 24 hours improves outcome from major trauma. J Trauma, 1999;47:964-969.

18. Lobo SM, Salgado PF, Castillo VG et al - Effects of maximizing oxygen delivery on morbidity and mortality in high-risk surgical patients. Crit Care Med, 2000;28:3396-3404.

19. Maillet JM, Le Besnerais P, Cantoni M et al - Frequency, risk factors, and outcome of hyperlactatemia after cardiac surgery. Chest, 2003;123:13611366.

20. Crowl AC, Young JS, Kahler DM et al - Occult hypoperfusion is associated with increased morbidity in patients undergoing early femur fracture fixation. J Trauma, 2000;48:260-267.

21. Smith I, Kumar P, Molloy S et al - Base excess and lactate as prognostic indicators for patients admitted to intensive care. Intensive Care Med, 2001;27:74-83.

22. Kirschenbaum LA, Astiz ME, Rackow EC - Interpretation of blood lactate concentrations in patients with sepsis. Lancet, 1998;352:(9132)921922

23. Leavy JA, Weil MH, Rackow EC - 'Lactate washout' following circulatory arrest. JAMA, 1988;260:662-664.

24. Giangiuliani G, Mancini A, Gui D - Validation of a severity of illness score (APACHE II) in a surgical intensive care unit. Intensive Care Med, 1989;15:519-522.

25. Buckley TA, Gomersall CD, Ramsay SJ - Validation of the multiple organ dysfunction (MOD) score in critically ill medical and surgical patients. Intensive Care Med, 2003;29:2216-2222.

26. Nguyen HB, Rivers EP, Knoblich BP et al - Early lactate clearance is associated with improved outcome in severe sepsis and septic shock. Crit Care Med, 2004;32:1637-1642.

27. Bakker J, Gris P, Coffernils M et al - Serial blood lactate levels can predict the development of multiple organ failure following septic shock. Am J Surg, 1996;171:221-226.

28. McNelis J, Marini CP, Jurkiewicz A et al - Prolonged lactate clearance is associated with increased mortality in the surgical intensive care unit. Am J Surg, 2001;182:481-485.

29. Singer M - Metabolic failure. Crit Care Med, 2005;33:(Suppl12):S539 S542. 